\title{
Editorial
}

\section{Recent Advances in Remote Spectral Sensing}

\author{
Chiman Kwan, ${ }^{1}$ Hairong Qi, ${ }^{2}$ and Trac $\operatorname{Tran}^{3}$ \\ ${ }^{1}$ Signal Processing, Inc., 9605 Medical Center Drive, Rockville, MD 20850, USA \\ ${ }^{2}$ The University of Tennessee, 304 Min H. Kao Building, 1520 Middle Drive, Knoxville, TN 37996, USA \\ ${ }^{3}$ The Johns Hopkins University, 3400 N. Charles Street, 215 Barton Hall, Baltimore, MD 21218, USA
}

Correspondence should be addressed to Chiman Kwan; chiman.kwan@signalpro.net

Received 11 July 2016; Accepted 12 July 2016

Copyright (c) 2016 Chiman Kwan et al. This is an open access article distributed under the Creative Commons Attribution License, which permits unrestricted use, distribution, and reproduction in any medium, provided the original work is properly cited.

Remote sensing and its applications have gained more and more attention from researchers in recent years. One clear indicator can be seen from the 2016 International Geoscience and Remote Sensing Symposium (IGARSS), which has received over 3,000 papers. This field is expanding and evolving rapidly. The aim of this special issue is an attempt to capture a small section of recent advances in remote sensing. We would like to thank all the contributing authors, reviewers, and journal staffs for making this special issue a reality. As will be seen shortly, the 6 papers indeed cover a wide range of remote sensing applications using airborne and space-borne instruments.

The paper by $\mathrm{H}$. Li et al. addressed an important problem in many state-of-the-art multispectral airborne imagers. Due to significant difference in intensity between different band images, image registration becomes very difficult even though the multispectral imager consists of identical monochrome cameras equipped with different bandpass filters. The authors proposed a two-stage image registration algorithm. The first stage is to use phase correlation method to calculate the parameters of a coarse-offset relationship between different band images. The second stage uses the scale invariant feature transform (SIFT) to detect the feature points. Actual experimental data were used to demonstrate the proposed algorithm. It was seen that conventional SIFTonly method failed whereas the proposed method can still achieve good registration performance.

Land cover land use (LCLU) classification is important for environment monitoring and urban planning. The paper by J. Jiao and Z. Deng focused on building and tree detection algorithms by using improved superpixels from large highresolution urban aerial images. The authors also proposed a method to calculate the tree parameters using a cost function and information from shadows. Experiments showed that their method is fast and robust, while still being simple and efficient, and they also indicate that the shadow is a good feature to estimate the tree height. The results of proposed algorithms have great potential for generating 3D urban models.

Airborne electromagnetic methods (AEM) systems are important for estimating abundance of natural resources. Typically, inductance is being measured using a rectangular loop onboard an aircraft. One serious problem with existing systems is that the effect of a finite-conducting ground on the inductance of the transmitting loop was neglected, or the ground was handled as a perfect conductor. In other words, there was no accurate method to evaluate ground's effect on the inductance of the transmitting loop. Consequently, the measurement will not be accurate. X. Jia et al. proposed a new and efficient algorithm to calculate ground's effect on the inductance of a rectangular loop. An experiment was constructed afield, showing that the inductance increased gradually when the loop was lifted up from $0 \mathrm{~m}$ to $30 \mathrm{~m}$, which supported the algorithm positively.

Using satellite data for generating vegetation profiles has the advantage of large area coverage. Due to the presence of clouds in the data, a composite 16-day period is normally used where at least a certain number of cloud free data are present. However, 16-day period vegetation profiles are less sensitive to real-time changes due to the composite period used for the bidirectional reflectance distribution function (BRDF) model. The paper by S. Kim et al. investigated the impact of different composite periods on the vegetation profiles. Geostationary Ocean Color Imager (GOCI) was 
the focus of their study. GOCI-BRDF-adjusted normalized difference vegetation indexes (NDVIs) with four different composite periods were compared with field-observation NDVI. The results showed that vegetation index seasonal profiles appeared similar to vegetation growth curves in both field observations from crop scans and GOCI NDVI data. The authors then concluded that a 12-day composite period was optimal in terms of BRDF simulation accuracy, surface coverage, and real-time sensitivity.

Information on the level of solar radiation can help many different applications, including analysis of the thermal load on buildings, solar energy collecting systems, crop growth models, and atmospheric energy balance studies. The paper by J.-M. Yeom et al. proposed an algorithm that generates the surface insolation mapping by integrating a physical model with the Communication, Ocean, and Meteorological Satellite (COMS) Meteorological Imager (MI) image. Original and topographically corrected solar radiation maps were created and their characteristics analyzed. It was concluded that the topographically corrected solar radiation map provided a better description of the actual surface geometric characteristics.

Biophysical parameters of vegetation canopies such as canopy chlorophyll content (CCC) and fraction of absorbed photosynthetically active radiation (FAPAR) provide important information in environmental studies concerning growth monitoring, stress detection, and yield estimation. They are very important inputs for models of gas exchange between terrestrial ecosystems and the atmosphere and for models of vegetation productivity. K. Sakowska et al. investigated the potential of using Sentinel-2 satellite for monitoring seasonal changes in grassland total CCC, FAPAR, and fraction of photosynthetically active radiation absorbed only by its photosynthesizing components (GFAPAR). Simulated Sentinel-2 satellite data using 2 years of ground based measurements were used in this study. It was concluded that Sentinel-2 satellite data has great potential in accurately retrieving biophysical parameters from space.

Chiman Kwan Hairong Qi Trac Tran 


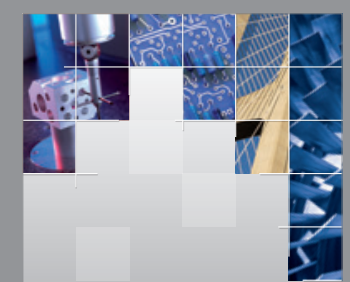

\section{Enfincering}
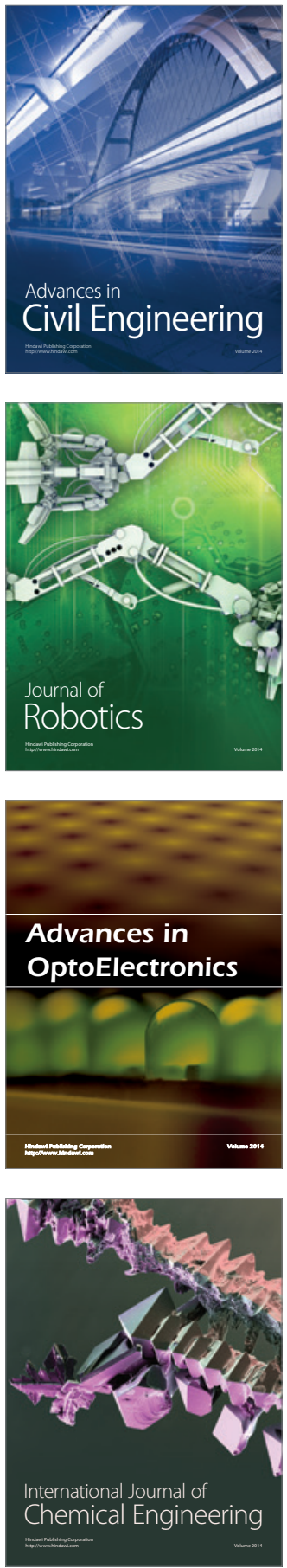

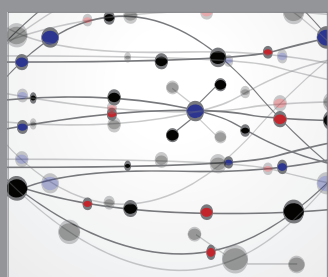

The Scientific World Journal

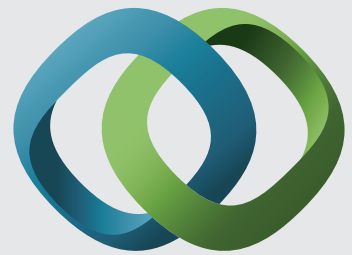

\section{Hindawi}

Submit your manuscripts at

http://www.hindawi.com
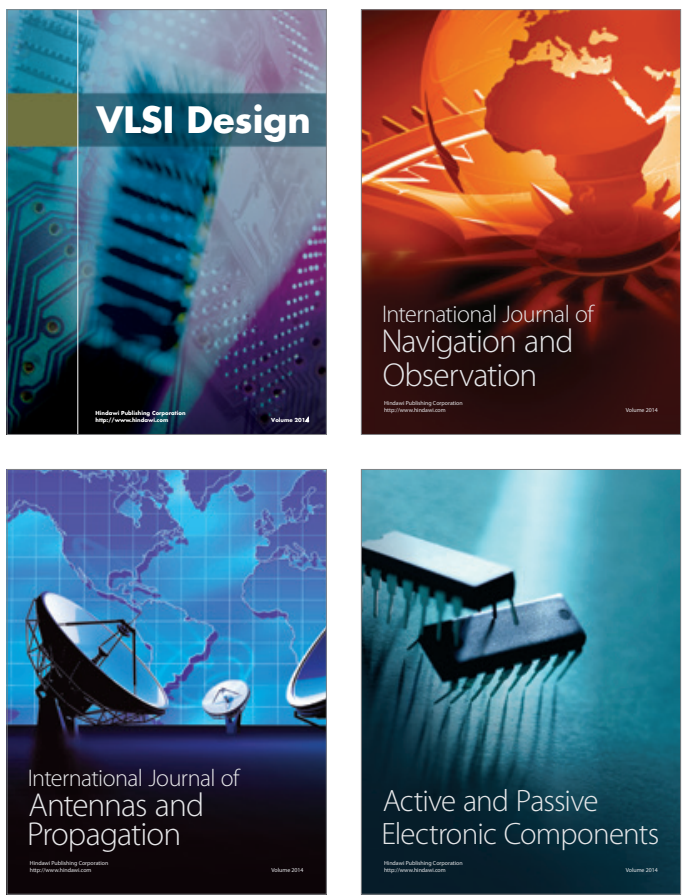
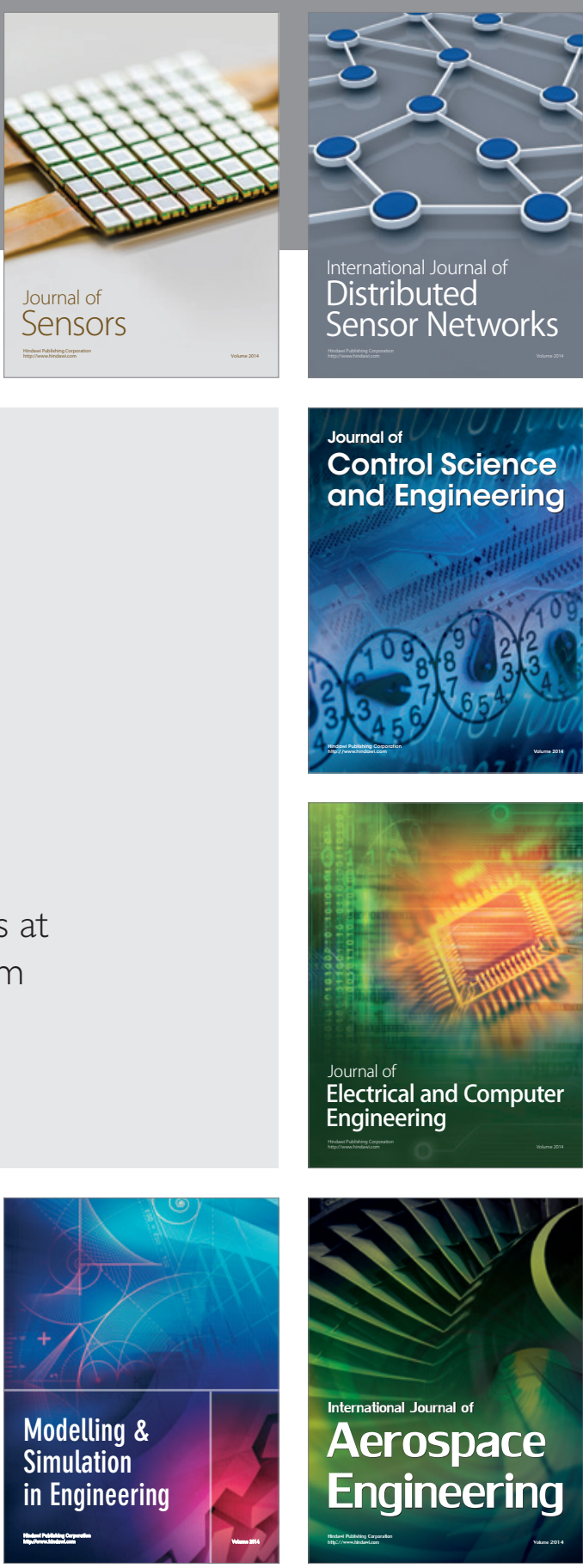

International Journal of

Distributed

Sensor Networks

Journal of

Control Science

and Engineering
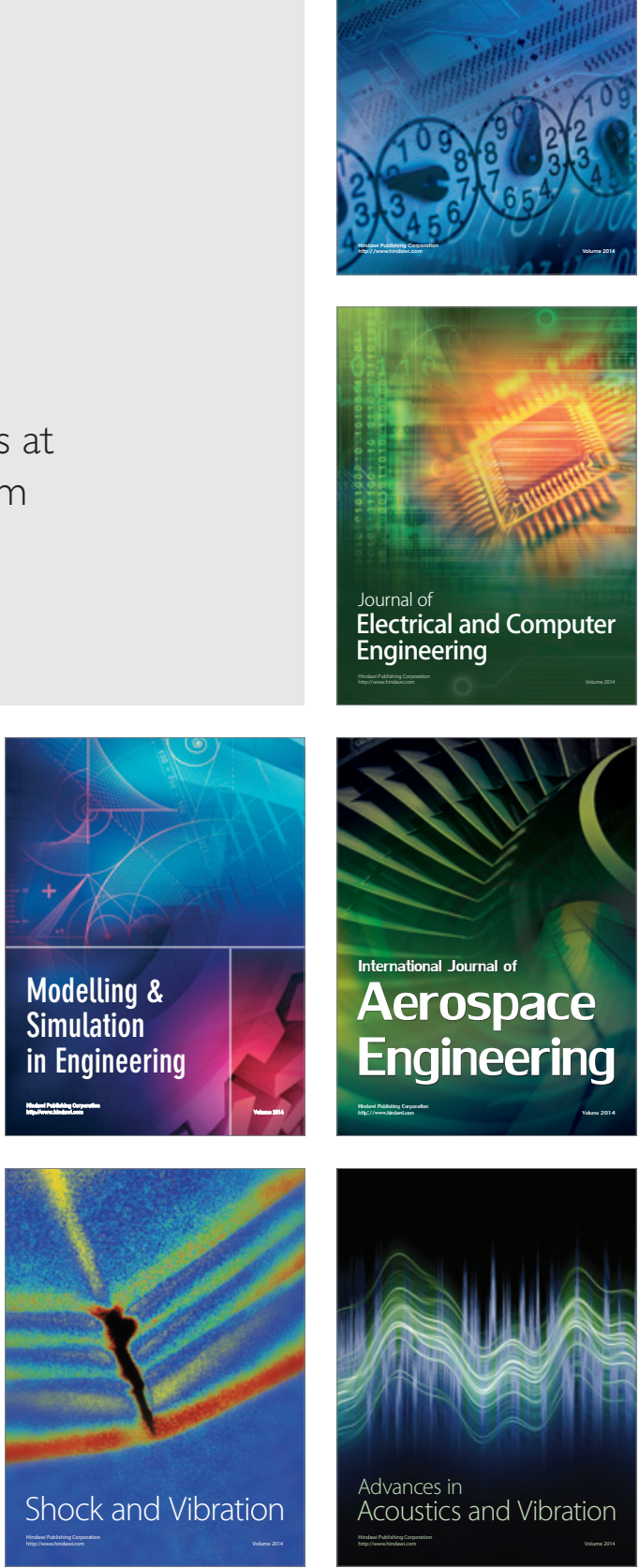